\title{
The technology of heat transfer enhancement in channels by means of flow pulsations
}

\author{
Anna Tsynaeva ${ }^{1, *}$, and Maxim Nikitin ${ }^{1}$ \\ ${ }^{1}$ Samara State Technical University, Institute of Architecture and Civil Engineering, \\ Molodogvardeyskaya str. 194, 443001 Samara, Russia
}

\begin{abstract}
The rate and efficiency of curing of concrete can boost when used intense heat. The work is dedicated to the development and research of technologies of intensification of heat transfer in channels by pulsations. The study was conducted by means of numerical methods based on mass and momentum conservation equations (Navier-Stokes) with software Code Saturne. Verification of implemented methods and software was performed. The research of heat transfer enhancement for semicircleshaped channel exposed to low-frequency pulsations was performed. The pulsation frequency of the flow during the study was in a range of $0 \ldots 10$ Hz. A significant (up to 4 times) increase of turbulent kinetic energy with implementing pulsations was detected. Flow pulsations with frequency of $10 \mathrm{~Hz}$ results in 1.21 times increase of heat transfer coefficient.
\end{abstract}

\section{Introduction}

Pulsations of the heat carrier flow are common for launch modes of thermal machines in the construction industry. In addition, the rate and efficiency of construction for monolithic building in winter conditions can be increased. This increase is the result of using thermal methods to accelerate concrete hardening. However, the efficiency of heat transfer should be high enough. Herewith the flow might be stabilized by means of controlled pulsations that are mainly used for heat transfer intensification [1,2]. However sustaining of such pulsations in channels of heat exchangers might be challenging due to damping effects of channels design.

In this regard an effect of channel design on heat transfer efficiency under flow pulsations should be determined. Bended channels should were considered in this study because they are commonly used in design of heat exchangers. The heat exchangers is basic element for heating of constructions of monolithic building. Since experimental research is very expensive authors used numerical simulations (finite volume method with an open source software Code Saturne) as a research tool [3].

\section{Numerical study}

\author{
Corresponding author: a.tsinaeva@gmail.com
}


Numerical study of heat transfer in bended channel (fig. 1) with pulsating inlet flow was conducted. Navier Stokes and conservation equations were closed with combined turbulence model (k-omega SST [4]). This model was successfully implemented in the previous numerical study of pulsations in linear channel [5]. An adequacy of implemented model was verified by comparison with adopted experimental data [6] for steady flow in a bended channel. Verification showed reasonable deviations from experimental data in a range of instrumental errors.
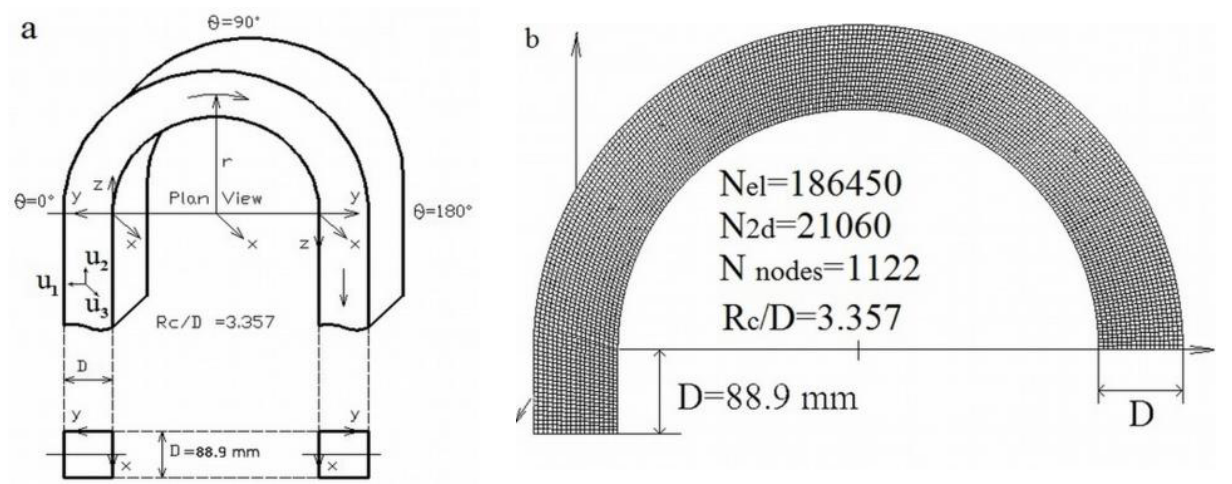

Fig. 1. Bended channel: $a$ - geometry, $b$ - mesh parameters.

Lateral symmetry of computational domain in respect to $\mathrm{YZ}$ plane was utilized to reduce computational effort. Computational mesh consisted of 186450 hexahedrons with prismatic layers providing $\mathrm{y}^{+}<1$.

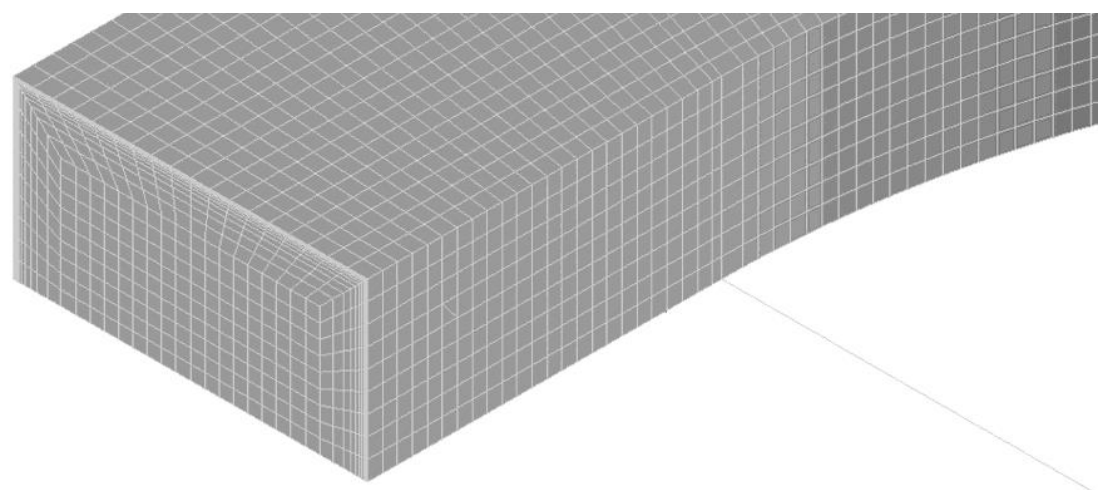

Fig. 2. Prismatic layers of computational mesh.

Gradients were calculated with a general method with handling of non-orthogonality, though mesh was explicitly orthogonal. Numerical algorithm which uses a combination of continuity and momentum equations to derive an equation for pressure wasn't applied for subsonic velocities. However transposed gradient and divergence source terms were included in momentum equation. Pressure relaxation of 0.5 was introduced to stabilize solution. Since there were no significant pressure gradients and head losses near outlet, the standard scheme of pressure interpolation was used for stratified flow. A semi-implicit algorithm (SIMPLEC) was used to speed up convergence.

Combined turbulence model (k-omega SST) was implemented since it appeared to be the most accurate during conducted verification [7]. Linear solvers precision was set at $10^{-8}$. 
However lowering threshold (down to $10^{-6}$ ) didn't show any significant improvement of adequacy during validation, but notably reduced computational effort.

Second order (Centered) discretization methods were used for solving hyperbolic partial differential equations for velocity and temperature fields. Auto switching to first order scheme (Upwind) was enabled for energy equation to stabilize solution during oscillations. Turbulence parameters ( $\mathrm{k}$ and omega) were calculated by first order linear solvers (Upwind). Further stabilization was achieved by temperatures range limitation $\left(0 \ldots 200{ }^{\circ} \mathrm{C}\right)$.

Simulation was conducted in transient mode since pressure and velocity oscillations observation was the major research objective. Constant time step of $0.0004 \mathrm{~s}$ was implemented to ensure Courant number to be less than 5 (max recommended for SIMPLEC algorithms). Moreover this time step had met requirements of minimum resolution for a quarter of period at maximum pulsations frequency. Overall $4 \mathrm{~s}$ of pulsating flow were modelled.

Air was used as a working media with constant thermophysical properties at $20{ }^{\circ} \mathrm{C}$. A constant heat flux $\left(q=1000 \mathrm{~W} / \mathrm{m}^{2}\right)$ was set at channel walls. Inlet velocity was defined with harmonic oscillations:

$$
u=\underline{u} \cdot \sin (2 \pi \tau)+\underline{u}
$$

where $\underline{u}$ - mean velocity, $\mathrm{m} / \mathrm{s} ; f$ - pulsations frequency, $\mathrm{Hz} ; \tau-$ time, $\mathrm{s}$.

Relative amplitude of oscillations was defined by (2) and was equal to 1.0 in this study.

$$
\beta=\left(\underline{u}_{\max }-\underline{u}_{\min }\right) /\left(\underline{u}_{\max }+\underline{u}_{\min }\right)
$$

Numerical study was conducted for the following frequencies of flow pulsation: $f=0$ $\mathrm{Hz}, f=5 \mathrm{~Hz}, f=10 \mathrm{~Hz}$. While Strouhal number was in range $\mathrm{Sh}=0 \ldots 1.67$. This range is consistent with quasi-steady and low-frequency pulsating flows, which were successfully simulated with RANS models before [5]. Harmonic variations of velocity are shown on fig. 3.

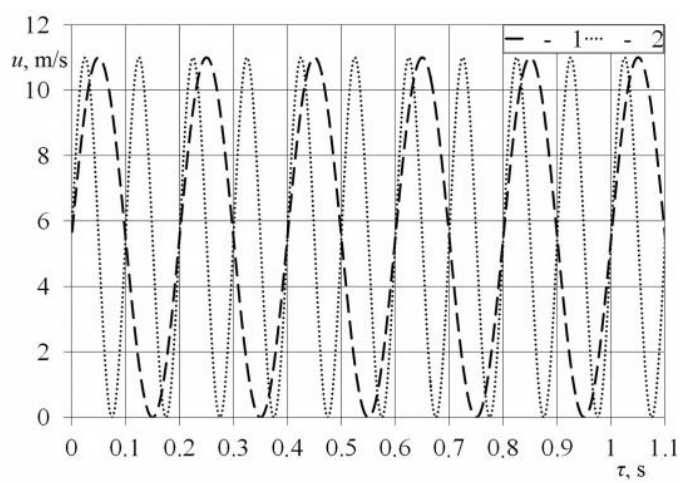

Fig. 3. Velocity variation: $1-f=5 \mathrm{~Hz} ; 2-f=10 \mathrm{~Hz}$.

Inlet flow velocity without pulsations was calculated as follows:

$$
\underline{u}_{s}=\underline{u}^{2} /\left(1+\beta^{2}\right)
$$

Other researches [8] indicate impact of flow pulsations on the flow structure, head losses and heat exchange. Therefore these impacts on flow and turbulence should be considered while conducting research of intensification of heat exchange in channels with pulsating flow. 


\section{Results and discussion}

Fig. 4 shows turbulence kinetic energy profile of pulsating flow at $\theta=90$ o. Depicted simulation data represent the acceleration stage of flow at $\tau \approx 1 \mathrm{~s}$. Results for steady flow without pulsations are also shown on fig. 4 for comparison.

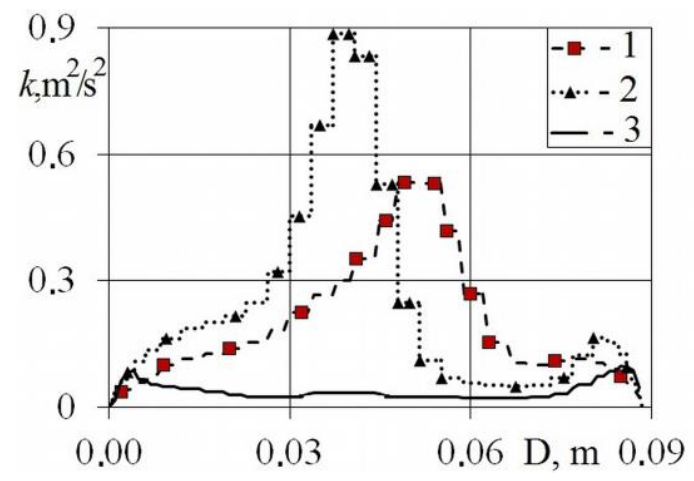

Fig. 4. Kinetic turbulence energy in lateral cross section at $\theta=90^{\circ}: 1-f=5 \mathrm{~Hz} ; 2-f=10 \mathrm{~Hz} ; 3-f$ $=0 \mathrm{~Hz}$.

Numerical simulation results (fig. 4) show increase of turbulent kinetic energy after pulsations application. Herewith at pulsations frequency of $5 \mathrm{~Hz}$ kinetic energy increases 1.8 times (at $y=0.09$ ) and 21.2 times (at $y=0.049$ ). For the flow, pulsating with frequency $10 \mathrm{~Hz}$, increase of turbulent kinetic energy can achieve 33.8 times compared to the steady flow.

To determine interdependency of flow pulsations and channel geometry dynamic of turbulence kinetic energy variation at the outlet at $\tau \approx 1 \mathrm{~s}$ should be analysed. This dependency is shown on fig. 5 .

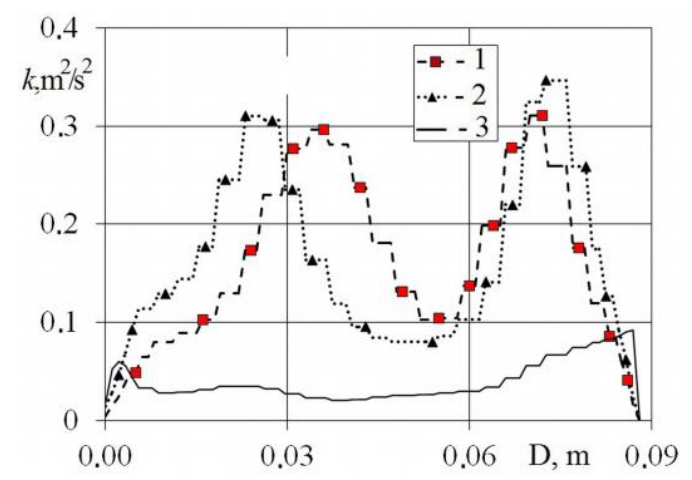

Fig. 5. Kinetic turbulence energy in lateral cross section at $\theta=180^{\circ}: 1-f=5 \mathrm{~Hz} ; 2-f=10 \mathrm{~Hz} ; 3-$ $f=0 \mathrm{~Hz}$.

Obtained results (fig. 5) are show that presence of pulsations affects turbulent kinetic energy distribution in computational domain. Moreover channel bending decreases turbulence (up to 3 times) and distorts mentioned distribution even more. At the same time introducing pulsations increases peak values of turbulent kinetic energy up to 4 times.

Fig. 6, a) represents velocity profile of channel flow in cross section at $\theta=180^{\circ}$ at time $\tau$ $\approx 1 \mathrm{~s}$ (during the stage of acceleration). 

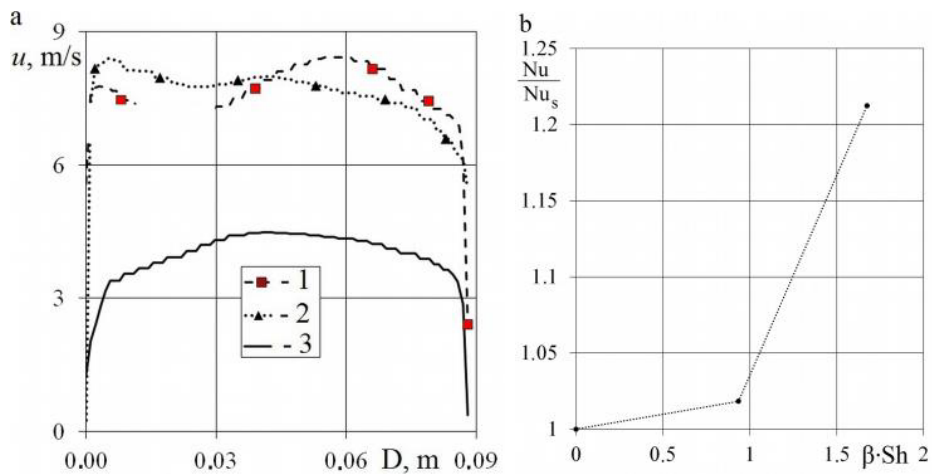

Fig. 6. Simulation results: a - velocity profile in cross section at $\theta=180^{\circ}: 1-f=5 \mathrm{~Hz} ; 2-f=10$ $\mathrm{Hz} ; 3-f=0 \mathrm{~Hz} ; \mathrm{b}$ - dependence of heat exchange rate from pulsations frequency.

From fig. 6, a) one can see that velocity profile of steady flow matches the developed turbulent flow. Velocity pulsations distort this profile based on its upstream structure. Fig. $6, b)$ represents simulation results summary for acceleration stage. Results mainly concern dependence of heat exchange rate from pulsations frequency.

\section{Conclusion}

Obtained results analysis confirmed strong dependency of heat transfer rate on pulsations frequency. For transient flow in dedicated range of parameters pulsations may increase heat transfer rate up to $21 \%$ compared to steady flow. This provides a sizable potential of gaining efficiency of operating heat exchangers by means of pulsating flow optimisation. This growth of heat transfer coefficient will allow to boost the heating of concrete structures. Consequently, this routine will reduced terms of erection of monolithic concrete buildings.

\section{References}

1. R. L. Webb, N. H. Kim, Principle of enhanced heat transfer (Taylor Francis, New York, 1994)

2. R. H. Keil, M. H. I. Baird, Ind. Eng. Chem. Process Des. Dev 10(4), 473-478 (1971)

3. Page of software Code Saturne, Information on http://code-saturne.org/cms/

4. Page of Langley Research Center: Turbulence Modeling. Information on http://codesaturne.org/cms/http://turbmodels.larc.nasa.gov/sst.html

5. T. Gebreegziabher, E. M. Sparrow, J. P. Abraham, E. Ayorinde, T. Singh, Numer. Heat Transfer 60(10), 811-826 (2011)

6. Y. D. Choi, C. Moon, S. H.Yang, Proc. of the International Symposium on Turbulence Modeling and Experiments (Dubrovnik, 1990)

7. A.A. Tsynaeva, M. N. Nikitin, Procedia Eng 150, 2340-2344 (2016)

8. A.E. Goltsman, I. A. Davletshin, N. I. Mikheev, A. A. Paereliy, Thermophys. Aeromech 22(3), 319-328 (2015) 